\title{
Effect of Intrapulmonary Inhalation of Honey, Nigella Sativa and Curcumin on Liver Function in Patients with Chronic Liver Disease
}

\author{
Ehab F. Mostafa, Amany M Ibrahim, Emad F. Hamed \\ Internal Medicine Department, Zagazig University, Egypt
}

\section{Corresponding Author Ehab F Mostafa}

Mobile:002012783487 85

E mail: hobanoh@yahoo.com

Received: 12 / $11 / 2012$ Accepted after revision: $29 / 11 / 2012$

Key words: Honey; Nigella Sativa; Curcumin; Inhalation; Liver Function; Chronic liver disease
Background and study aim: Evidence indicates, that honey, nigella sativa and Curcumin can exert several healthbeneficial effects. The aim of this study was to examine the efficacy and safety of honey, curcumin and nigella sativa inhalation on liver function in patients with chronic liver disease.

Patients Methods:The study was conducted in 58 Patients (group1) with chronic liver disease due to hepatitis $\mathrm{C}$ and or $\mathrm{B}$ infection, with abnormal liver function test regarding liver enzymes, serum albumin, serum total bilirubin and international normalized ratio (INR). Another 43 patients(group2) with chronic liver disease with abnormal liver functions test as a control diseased subjects.

Patients were subjected to inhalation of honey solution diluted with water with total $5 \mathrm{ml}$ solution with $1 \mathrm{ml}$ of curcumin solution, then adding of 2 drops of nigella sativa oil, the patients subjected to 2 times inhalation per day for 3 months. The other 43 control subjects received inhalation of $10 \%$ dextrose for the same duration.
Results: there was significant difference in patients group before and after mixed solution inhalation for 3 months with decrease in serum level of ALT and less significant decrease in AST, also there was significant decrease in serum total bilirubin and INR level, already with significant increase in serum albumin. Correlation between duration of exposure to the mixed solution inhalation and change of liver functions after one and 3 months duration, showed significant negative correlation between duration and decrease in ALT, AST, INR and serum bilirubin with positive correlation with serum albumin. No significant changes was detected in liver functions in control subjects after inhalation of $10 \%$ dextrose for the same duration.

Conclusion: Natural medications like honey, curcumin and nigella sativa administered by new methods like inhalation my contribute in improving liver functions with high safety profile.

\section{INTRODUCTION}

Honey has been an ingredient of traditional medicine on account of its dietary and curative properties since ancient times .

Starting in the early 1970 researchers from different scientific fields have investigated the chemical and biological properties of honey, including antibacterial, bacteriostatic, anti-inflammatory, wound and sunburn healing activities .
Recent views propose honey not only as health promoting dietary supplement, but shed light on antioxidant, non-peroxide dependent properties [1].Evidence indicates that honey can exert several healthbeneficial effects such as gastroprotective [2], hepatoprotective [3], reproductive[4,5], hypoglycemic[6], antioxidant[6], antihypertensive[7], antibacterial[8], anti-fungal [9] and antiinflammatory[10] effects. 
Honey also contains other bioactive constituents such as organic acids, ascorbic acid, trace elements, vitamins, amino acids, proteins and Maillard reaction products[11]. The data presented suggest that honey, administered alone or in combination with conventional therapy, might be of therapeutic benefits in the management of chronic diseases commonly associated with oxidative stress.

Considering that the bulk of these data emanate from animal studies, it is worthwhile to perform clinical studies that investigate if this antioxidant effect of honey can be extrapolated to human subjects with chronic diseases. The liver plays an important role in many metabolic processes such as glycemic control, detoxification of xenobiotics, synthesis of lipoproteins, hormones and enzymes[12]. Available evidence suggests that the liver is susceptible to oxidative stress and damage; and the beneficial effect of antioxidants on hepatic oxidative stress has been documented[13].

The amelioration of oxidative stress, as a result of honey administration, was accompanied by significant reductions in the size of enlarged hepatocytes and edema, restoration of bile canaliculi dilatation and reduced number of apoptotic cells[14].

Similar hepatoprotective effect of honey was also reported in rats with obstruction of the common bile duct[15], in rats with N-ethylmaleimide (NEM)-induced liver injury, honey supplementation significantly restored the levels of hepatic glutathione, ameliorated the (NEM)induced congestion and mononuclear cell infiltration in the liver[16].

These findings, generally, suggest that amelioration of oxidative stress in the liver may contribute to the hepatoprotective effect of honey.

Nigella sativa ( $N$. sativa) is a herbaceous plant used as a natural food additive. It belongs to the botanical family of Ranunculaceae, grows in the Middle East, Central Europe and Western Asia. Traditionally these seeds are used for the prevention and cure of many ailments for over 2000 years. The principal active ingredient isolated from the volatile oil of $N$. sativa is thymoquinone (TQ), it has been reported that TQ exhibits many pharmacological effects, including antioxidant and protective effects against hepatotoxins[17].Recently conducted clinical and experimental researches have shown many therapeutic effects of NS extracts such as immunomodulator, anti-inflammatory and antitumour agents[18].

Since $1900 \mathrm{bc}$, several therapeutic activities have been attributed to the rhizomes of the plant Curcuma longa for a variety of diseases, including liver disorders. Curcumin, the main active compound obtained from this plant, was first isolated two centuries ago and its structure as diferuloylmethane was determined in 1910 . Curcumin has shown anti-inflammatory, antioxidant, antifungal, antibacterial and anticancer activities. The pharmacological properties of curcumin were reviewed recently and focused mainly on its anticancer properties. However, its beneficial activity on liver diseases (known centuries ago, and demonstrated recently utilizing animal models) has not being reviewed in depth until now. The curcumin ability to inhibit several factors like nuclear factor-kappa B, which modulates several pro-inflammatory and profibrotic cytokines as well as its antioxidant properties [19].

An increasing number of medications are being administered by inhalation. However, proper dosing, frequency, formulation, and the optimal delivery device remain to be determined for many of these agents. Inhalation therapy has many advantages compared with other routes of administration including achieving a high drug concentration in the lung, lack of systemic adverse effects, ease of administration, and patient convenience. A broad range of patients may benefit from this type of drug delivery[20].

Aerosolized medications is better than oral route because within this route nearly most of the medication is delivered to the blood without changes in contrast to oral route, the medications subjected to different factors through the gastrointestinal tract affecting it.

Aerosolized medications may be delivered to the lower airway either through the nasal cavity or the oropharynx, however, the oropharynx route is preferable for several reasons. First, the alveolar region of the lung provides a larger surface area for drug absorption (approximately $75 \mathrm{sq} \mathrm{m}$ ) compared to intranasal route[21]. Alveolar walls are thin and well perfused, allowing rapid drug absorption. The large surface area coupled with the high amount of blood flow through the pulmonary tissues maximizes drug absorption. Second, pulmonary mucociliary clearance 
mechanisms are minimal in the alveolar region of the lungs. Unlike the terminal portions of the lung, however, the mucociliary clearance of the nasal passages are much more effective[22].

Thus, intrapulmonary administration is associated with less rapid elimination of medications compared to intranasal, allowing prolonged deposition time and, for some medications, subsequent systemic absorption. For these reasons, intrapulmonary inhalation seems to be emerging as the optimal route for administration of aerosolized solutions.

In this study, we investigated the effect of honey, curcumin and nigella sativa inhalation on liver function in patients with chronic liver disease.

\section{PATIENTS AND METHODS}

After approval from the medical ethical committee, this study was conducted from March to August 2012, the study was conducted in 58 patients (group 1) selected from outpatients of Zagazig university hospital with chronic liver disease due to hepatitis $\mathrm{C}$ and or $\mathrm{B}$ infection already with abnormal liver function test regarding liver enzymes, serum albumin, serum total bilirubin and international normalized ratio INR. Another 43 patients (group 2) with chronic liver disease with abnormal liver functions test as a diseased control subjects.

Patients were subjected to detailed medical history, thorough physical examination, liver function tests (ALT,AST, INR, serum albumin and bilirubin), renal function tests (urea and creatinine), pulmonary function tests(FEV1, FVC, FEV1 IFVC ratio) using spirometry were done directly befor the study and at one and 3 months latter., abdominal ultrasound, chest $\mathrm{X}$ ray.

Inhalation of suspension containing honey solution diluted with water $(60 \mid 40)$ [7] with total $5 \mathrm{ml}$ solution with $1 \mathrm{ml}$ of curcumin solution prepared by adding $50 \mathrm{gm}$ of curcumin powder dissolved in $50(\mathrm{ml})$ water with adding one sodium bicarbonate tablet of $325 \mathrm{mg}$ to enhance dissolving then filtering all to get clear fluid, then adding of 2 drops of nigella sativa oil which purchased from the market. The honey used is a cotton flower honey; the inhalation device used nebulizer in unit dose presentation to prevent microbial contamination during use, the patients subjected to 2 times inhalation per day for 3 months. These preparations are based on a systematic review of scientific literature edited and peer-reviewed by contributors to the Natural Standard Research Collaboration (www.naturalstandard.com).

Any patients showed irritation or change in pulmonary function tests will be excluded, but no one did.

The control group received inhalation of $10 \%$ dextrose $5 \mathrm{ml}$ for the same duration.

Data collected after one month and 3 months later.

All subjects included in this study were informed to stop any medication that may affect liver function before the study.

\section{Statistical analysis}

The data were presented as means \pm standard errors. For the comparison of statistical significance between two groups paired sample ttest was used. Values were accepted as being statistically significant if a $\mathrm{P}$ value was less than 0.01. Correlation between duration of exposure to the mixed solution inhalation and change of liver function was done using Pearson correlation.

\section{RESULTS}

As shown in Table 1 there was no significant changes in both groups. Table 2 showed significant difference in patients group before and after mixed solution inhalation for 3 months with decrease in serum level of ALT and less significant decrease in AST, also there was significant decrease in serum total bilirubin and INR level, already with significant increase in serum albumin, no significant difference in control patients group 2 before and after 3 months of dextrose inhalation Table 3.

Table 4 showed Correlation between duration of exposure to the mixed solution inhalation and change of liver functions after one and 3 months duration, with significant negative correlation between duration and decrease in ALT, AST, INR and serum bilirubin with positive correlation with serum albumin.

No significant changes was detected in liver functions in control subjects after inhalation of $10 \%$ dextrose for the same duration. 
Table (1): Patients and control subjects characteristics (mean \pm SD)

\begin{tabular}{|l|c|c|c|}
\hline & Group 1 $(\boldsymbol{n}=\mathbf{5 8})$ & Group 2 $(\boldsymbol{n}=\mathbf{4 3})$ & $\boldsymbol{P}$ \\
\hline Age (yr) & $48 \pm 4$ & $46 \pm 4$ & NS \\
\hline Serum creatinine (mg/dL) & $1.2 \pm 0.2$ & $1.0 \pm 0.1$ & NS \\
\hline BUN (mg/dL) & $25.5 \pm 7.3$ & $23.5 \pm 1.2$ & NS \\
\hline AST (U/L) & $65 \pm 8.9$ & $63 \pm 8.2$ & NS \\
\hline ALT (U/L) & $59 \pm 14$ & $61 \pm 14.1$ & NS \\
\hline Total bilirubin (mg/dL) & $2.7 \pm 0.5$ & $2.8 \pm 0.6$ & NS \\
\hline Serum albumin (g/Dl) & $2.3 \pm 0.44$ & $2.4 \pm 0.46$ & NS \\
\hline INR & $1.6 \pm 0.2$ & $1.5 \pm 0.1$ & NS \\
\hline
\end{tabular}

NS=non-significant

Table (2): Mean difference \pm SD in patients of group 1 before and after 3 months of mixed solution inhalation.

\begin{tabular}{|l|c|c|c|c|}
\hline & Before & After & t & $\boldsymbol{P}$ \\
\hline ALT(U/L) & $59 \pm 14$ & $38 \pm 8$ & 8.9 & 0.001 \\
\hline AST (U/L) & $65 \pm 8.9$ & $55 \pm 9$ & 4.8 & 0.004 \\
\hline Serum albumin (g/dl) & $2.2 \pm 0.4$ & $3 \pm 0.8$ & -6.2 & 0.001 \\
\hline Total bilirubin (mg/dL) & $2.7 \pm 0.5$ & $1.7 \pm 0.3$ & 7.7 & 0.001 \\
\hline INR & $1.6 \pm 0.2$ & $1.3 \pm 0.1$ & 6.6 & 0.001 \\
\hline
\end{tabular}

Table (3): Mean difference $\pm \mathrm{SD}$ in control patients group 2 before and after 3 months of dextrose inhalation.

\begin{tabular}{|l|c|c|c|}
\hline & Before & After & $\boldsymbol{P}$ \\
\hline ALT (U/L) & $61 \pm 14.1$ & $63 \pm 13.6$ & NS \\
\hline AST (U/L) & $63 \pm 8.2$ & $61 \pm 7.8$ & NS \\
\hline Serum albumin (g/dl) & $2.4 \pm 0.46$ & $2.3 \pm 0.38$ & NS \\
\hline Total bilirubin $(\mathrm{mg} / \mathrm{dL})$ & $2.8 \pm 0.6$ & $2.7 \pm 0.5$ & NS \\
\hline INR & $1.5 \pm 0.1$ & $1.6 \pm 0.3$ & NS \\
\hline
\end{tabular}

Table (4): Correlation between duration of exposure to inhalation of the mixed solution and change of liver function.

\begin{tabular}{|l|c|c|c|c|}
\hline & $\begin{array}{c}\text { Mean }(\boldsymbol{n}=\mathbf{5 8}) \\
\text { After one month }\end{array}$ & $\begin{array}{c}\text { Mean }(\boldsymbol{n}=\mathbf{5 8}) \text { After } \\
3 \text { months }\end{array}$ & Correlation & $\boldsymbol{P}$ \\
\hline ALT (U/L) & $44 \pm 10$ & $38 \pm 8$ & -.731 & 0.001 \\
\hline AST (U/L) & $60 \pm 10$ & $55 \pm 9$ & -.641 & 0.001 \\
\hline Total bilirubin (mg/dL) & $2 \pm 0.4$ & $1.7 \pm 0.3$ & -.531 & 0.001 \\
\hline INR & $1.4 \pm 0.2$ & $1.3 \pm 0.1$ & -.481 & 0.001 \\
\hline Serum albumin (g/dL) & $2.7 \pm 0.5$ & $3 \pm 0.8$ & +.561 & 0.001 \\
\hline
\end{tabular}

No significant changes in pulmonary functions tests regarding FVC,FEV1,FEV1|FVC ratio.

\section{DISCUSSION}

Honey is a natural antioxidant which may contain flavinoids, ascorbic acid, tocopherols, catalase, and phenolic compounds all of which work together to provide a synergistic antioxidant effect, scavenging and eliminating free radicals[23].

Nigella Sativa treatment decreased the elevated liver enzyme levels and also increased the reduced antioxidant enzyme levels in patients with hepatitis. Previously performed clinical and experimental investigations have shown that NS has a protective effect against oxidative damage in infected hepatocytes. It was found that the fixed oil of NS has both antioxidant and antieicosanoid effects greater than thymoquinone which is its active constituent [13]. Furthermore, NS has antioxidant activity by suppressing the chemiluminescence in phagocytes[24].

Curcumin has shown pleiotropic beneficial effects in many iatrogenic organ maladies. It apparently ameliorated the gross and histological alterations in hepatocytes in patients with 
hepatitis. It decreased the relative liver/ body weight ratio. It also mitigated pleomorphism, apoptotic bodies, necrosis and inflammation as well as dilatation of central lobular sinusoid. It improved fatty changes with minimal microvesicular steatosis and hepatocytes became more granular. Recently, the protective effect of curcumin on rat liver injury induced by $\mathrm{CCl} 4$ was demonstrated by $\mathrm{Fu}$ et al. who showed that curcumin administration prevented ALT and AST increases and improved liver function in CCl4-induced liver damage[25].

Our result showed that intrapulmonary administration of honey, curcumin and nigella sativa solution did not cause any adverse effect in all patients receiving this mixed solution, already with improvement in liver enzymes with significant reduction in ALT and AST. Also our results showed significant improvement of INR, serum bilirubin and serum albumin level.

We noticed that there was more improvement in liver functions with increasing period of treatment reaching to 3 months as shown in table 4. In which there was positive correlation between improvement in liver functions and period of exposure to the mixed solution.

Our results are in agreement with Antony et al., who reported that honey has a hepatoprotective activity, it was found that honey reduce lipid peroxidation and nitric oxide and greatly improved liver enzymes, referred to its antioxidant activity [26]. Honey have a hepatoprotective activity against methyl nitrosourea (MNU)-induced oxidative stress and inflammatory response by keeping normal defense system and decrease NO [27]. Also honey supplementation significantly restored the levels of hepatic glutathione, ameliorated the (NEM)-induced congestion and mononuclear cell infiltration in the liver.

Improvement of serum bilirubin may be correlated with Kilicoglu et al, who reported that amelioration of oxidative stress, as a result of honey administration, was accompanied by significant reductions in the size of enlarged hepatocytes and edema, restoration of bile canaliculi dilatation and reduced number of apoptotic cells with subsequent decrease in serum bilirubin[14].

Improvement of liver function by adding NS is documented by reports from Turkdogan et al. who, observed that Nigella Sativa has a significant hepatoprotective effect in $\mathrm{CCl}_{4}{ }^{-}$ administrated rabbits, and the hepatocellular degenerative and necrotic changes are slight in NS-treated group and also they found that NS can prevent liver fibrosis and cirrhosis, suggesting that NS protects liver against fibrosis possibly through immunomodulator and antioxidant activities[28].

Curcumin inhibits nuclear factor-kappa B (NF_B) binding activity and the expression of tumor necrosis factor (TNF), interleukin (IL-12), monocyte chemoattractant protein-1 (MCP-1), macrophage inhibitory protein (MIP-2), Cyclooxygenase-2 (COX-2), and nitric oxide (iNOS) in endotoxin treated Kupffer cells, which supports our results in the reduction of liver enzymes which may be consider as markers of liver inflammation, this was proved by Amin et al, who treated Isolated Kupffer cells with vehicle or the indicated concentrations of curcumin for $1 \mathrm{~h}$ before treatment with endotoxin lipopolysccharides $(100 \mathrm{ng} / \mathrm{ml})$ and found that treatment with curcumin inhibited NF_B binding activity and the expression of TNF_, IL-12, MCP-1, MIP-2, COX-2, and iNOS in endotoxin treated cells [29].

Conclusion: Natural medications like honey, curcumin and nigella sativa administered by new methods like inhalation my contribute in improving liver functions with high safety profile.

Recommendation: Such studies have to be applied for a larger numbers of patients with a longer period and study if such medications can affect hepatitis B or C viral load.

\section{Funding: None}

Conflicts of interest: The authors declare that there is no conflict of interest.

Ethical consideration: Informed consents were obtained from all patient. The study was performed according to the ethical standards for human experimentation and was approved by the scientific committee of Faculty of Medicine, Zagazig University.

\section{REFERENCES}

1- Beretta G, Granata P, Ferrero M, Orioli M, Facino R. "Standardization of antioxidant properties of honey by a combination of spectrophotometric / fluorimetric assays and chemometrics". Analytica Chimica Acta 2005; 533: 185-191. 
2- Gharzouli K, Amira S, Gharzouli A, Khennouf S. Gastroprotective effects of honey and glucosefructose-sucrose-maltose mixture against ethanol, indomethacin- and acidified aspirin-induced lesions in the rat. Exp. Toxicol. Pathol. 2002; 54: 217-221.

3- Al-Waili NS, Saloom KY, Al-Waili TN, Al-Waili AN, Akmal M, Al-Waili FS et al .Influence of various diet regimens on deterioration of hepatic function and hematological parameters following carbon tetrachloride: A potential protective role of natural honey. Nat. Prod. Res. 2006; 20: 1258 1264.

4- Mohamed M, Sulaiman SA, Jaafar H, Sirajudeen $\mathrm{KN}$. Effect of different doses of Malaysian honey on reproductive parameters in adult male rats. Andrologia 2012;44 Suppl 1:182-186.

5- Zaid SS, Sulaiman SA, Sirajudeen KN, Othman NH. The effects of Tualang honey on female reproductive organs, tibia bone and hormonal profile in ovariectomised rats-animal model for menopause. BMC Complement. Altern. Med. $2011 ; 10: 82$.

6- Erejuwa OO, Gurtu S, Sulaiman SA, Ab Wahab MS, Sirajudeen KN, Salleh MS. Hypoglycemic and antioxidant effects of honey supplementation in streptozotocin-induced diabetic rats. Int. J. Vitam. Nutr. Res. 2010; 80: 74-82.

7- Al-Waili N. Intrapulmonary administration of natural honey solution, hyperosmolar dextrose or hypoosmolar distill water to normal individuals and to patients with type- 2 diabetes mellitus or hypertension: Their effects on blood glucose level, plasma insulin and C-peptide, blood pressure and peaked expiratory flow rate. Eur. $J$. Med. Res. 2003; 8:295-303.

8- Tan HT, Rahman RA, Gan SH, Halim AS, Hassan SA, Sulaiman $S$ et al. The antibacterial properties of Malaysian tualang honey against wound and enteric microorganisms in comparison to manuka honey. BMC Complement. Altern. Med. 2009; 9: 34.

9- Koc AN, Silici S, Kasap F, Hormet-Oz HT, Mavus-Buldu H, Ercal BD. Antifungal activity of the honeybee products against Candida spp. and Trichosporon spp. J. Med. Food. 2011; 14: 128134.

10- Kassim M, Achoui M, Mustafa MR, Mohd MA, Yusoff KM. Ellagic acid, phenolic acids and flavonoids in Malaysian honey extracts demonstrate in vitro anti-inflammatory activity. Nutr. Res. 2010; 30: 650-659.

12- Klip A, Vranic M. Muscle, liver and pancreas: Three Musketeers fighting to control glycemia. Am. J. Physiol. Endocrinol. Metab 2006; 291: E1141-E1143.
13- Dias AS, Porawski M, Alonso M, Marroni N, Collado PS, Gonzalez-Gallego J. Quercetin decreases oxidative stress, NF-kappaB activation and iNOS overexpression in liver of streptozotocin-induced diabetic rats. J. Nutr 2005; 135: 2299-2304.

14- Kilicoglu B, Gencay C, Kismet K, Serin Kilicoglu S, Erguder I, Erel $S$ et al The ultrastructural research of liver in experimental obstructive jaundice and effect of honey. Am. J. Surg 2008; 195: 249-256.

15- Erguder BI, Kilicoglu SS, Namuslu M, Kilicoglu B, Devrim E, Kismet K et al. Honey prevents hepatic damage induced by obstruction of the common bile duct. World J. Gastroenterol 2008; 14:3729-3732.

16- Korkmaz A, Kolankaya D. Anzer honey prevents N-ethylmaleimide-induced liver damage in rats. Exp Toxicol Pathol 2009; 61: 333-337.

17- Al-Ghamdi MS. Protective effect of Nigella sativa seeds against carbon tetrachloride-induced liver damage. Am J Chin Med 2003;31(5):721-8.

18- Mehmet K, Omer C, Mustafa B. Hepatoprotective effects of Nigella sativa $\mathrm{L}$ and Urtica dioica $\mathrm{L}$ on lipid peroxidation, antioxidant enzyme systems and liver enzymes in carbon tetrachloride-treated rats. World J Gastroenterol 2005; 14;11(42): 6684-6688.

19- Rivera-Espinoza, Y, Muriel P. Pharmacological actions of curcumin in liver diseases or damage. Liver Int. 2009; 29(10):1457-66.

20- Bayat, M, Cook AM. Intrapulmonary administration of medications. J Neurosci Nurs 2004;36(4):231-5.

21- Laube BL. Treating diabetes with aesolized insulin. Chest 2001; 120: 99s-106s.

22- Illum, L. Nasal drug delivery--Possibilities, problems and solutions. Journal of Controlled Release 2003; 87: 187-198.

23-Johnston J, Sepe H, Miano C, Brannan R, Alderton A. "Honey inhibits lipid oxidation in ready-to-eat ground beef patties". Meat Science 2005; $70: 627-631$.

24- Haq A, Abdullatif M, Lobo PI, Khabar KS, Sheth KV, al-Sedairy ST. Nigella sativa: effect on human lymphocytes and polymorphonuclear leukocyte phagocytic activity. Immunopharmacology 1995; 30: 147-155.

25- Ramadan AM, OMar M. Curcumin Attenuates Methotraxate-Induced Hepatic Oxidative Damage in Rats. Journal of the Egyptian Nat. Cancer Inst 2008; 20,(2): 141-148. 
26- Antony SM, Rieck J, Dawson R. Effect of dry honey on oxidation in turkey breast meat. Poult. Sci.2000;79: 1846-1850.

27- Mabrouk GM, Zohny G, Ali E, Ismail E, Moselhy S. Bee honey and Nigella sativa inhibit nitric oxide mediated cytochrome $\mathrm{C}$ release and down-regulation of connex in 43 induced by methyl nitrosourea in hepatic tissues of sprague dawely rats. Egypt J. Biochem 2004; 22: 73-87.
28- Turkdogan MK, Ozbek H, Yener Z, Tuncer I, Uygan I, Ceylan E. The role of Urtica dioica and Nigella sativa in the prevention of carbon tetrachloride-induced hepatotoxicity in rats. Phytother Res 2003; 17: 942-946.

29-Amin A, Kalle J, George L, Amir R, Peter T, Andrew J. Curcumin prevents alcohol-induced liver disease in rats by inhibiting the expression of NF-_B-dependent genes. Am $J$ Physiol Gastrointest Liver Physiol. 200;3 284: G321G327. 